\title{
Analysis of the mechanical properties of a reinforced thermoplastic pipe (RTP)
}

\author{
M.P. Kruijer*, L.L. Warnet, R. Akkerman \\ Section of Applied Mechanics and Polymers, Department of Mechanical Engineering, University of Twente, \\ P.O. Box 217, 7500 AE, Enschede, The Netherlands
}

\begin{abstract}
This paper describes the analysis of a long length reinforced thermoplastic pipe. For this new class of pipe, which is constructed of a polyethylene liner pipe over wrapped with two layers of non-impregnated twisted aramid cords, peculiar deformation behaviour was observed when a pipe was pressurised. This behaviour was found to be a result of a difference in cord-slack between the two reinforcement layers in conjunction with an unbalanced torsion moment generated by the two reinforcing layers. Cord slack is a certain surplus length of the reinforcing cords relative to the pipe geometry. The cord-slack is estimated for the two different layers and first incorporated into an earlier published model, based on a plane stress characterisation. As no substantial improvement has been achieved by this approach, a new model based on a plane strain characterisation is introduced. This model shows good agreement with the experimentally determined strains for the hydrostatic pressure load-case.
\end{abstract}

(C) 2004 Elsevier Ltd. All rights reserved.

Keywords: A. Thermoplastic resin; B. Mechanical properties; D. Mechanical testing; On-shore oil industry composite pipe

\section{Introduction}

Composite pipes are increasingly used in the oil and gas industry, instead of conventional carbon steel pipes. The main reason is the better corrosion resistance of composite pipes compared to steel pipes. The first composite alternatives to steel pipelines consisted of composite pipes based on thermoset matrices. The properties of thermoset composites are well known and documented [1]. However, the increasing demands of the oil and gas industry, involving high temperature resistance and the need for damage tolerance and flexibility, often exceed the capabilities of thermosets. As thermoplastics can satisfy these demands, several types of continuous fibre reinforced thermoplastic pipe (RTP) systems are currently under development.

The principal components of an RTP are:

- A thermoplastic liner, the major function being to provide a leak free and corrosion resistant containment for the transported fluid.

\footnotetext{
* Corresponding author. Fax: +31-228-355540.

E-mail address: m.p.kruijer@ctw.utwente.nl (M.P. Kruijer).
}

- An even number of helical windings of continuous reinforcement to contain the applied inner pressure and other loads.

- An outer cover layer to protect the fibre layers from external damage.

As mentioned in Ref. [2], the continuous reinforcement material usually consists of carbon, aramid or glass fibres, embedded in a thermoplastic matrix of polyethylene (PE), polypropylene (PP), polyamide (PA) or polyvinylidenefluoride (PVDF). The fibres can be either single filaments, yarns or braided. As discussed in Ref. [3], RTPs can be manufactured using various types of helical winding processes. One of these is the helical tape wrapping method. This process, which will be discussed in more detail in Section 2.2, has the potential to be continuous (as opposed to thermoplastic filament winding), which enables the production of substantially long pipes.

The pipes considered here are produced by the helical tape wrapping method, using prepreg tapes in which non-impregnated twisted aramid yarns are embedded in a high-density PE matrix. Since the twisted aramid yarns are not impregnated, there is no bonding between the fibres 


\begin{tabular}{|c|c|c|c|}
\hline \multicolumn{4}{|l|}{ Nomenclature } \\
\hline$A_{\mathrm{f}}$ & $\begin{array}{l}\text { total cross-section surface of the } \\
\text { reinforcement tape }\end{array}$ & $\varepsilon_{11}, \varepsilon_{22}, \varepsilon_{12}$ & $\begin{array}{l}\text { strains in local directions (i.e. cord and } \\
\text { matrix directions) }\end{array}$ \\
\hline$A_{\mathrm{m}}$ & cross-section surface of the matrix & $\sigma_{r r}, \sigma_{\theta \theta}, \sigma_{z z}, \sigma_{z \theta}$ & stresses in global directions \\
\hline & material & $\sigma_{11}, \sigma_{22}, \sigma_{12}$ & stresses in local directions (i.e. cord and \\
\hline$A_{\mathrm{c}}$ & cross-section surface of the cords & & matrix directions) \\
\hline$V_{\mathrm{f}}$ & fibre volume fraction & $A_{1}, B_{1}, C_{1}$ & unknown constants in the plane stress \\
\hline$V_{\mathrm{m}}$ & matrix volume fraction & & $\begin{array}{l}\text { model } \\
\text { unknown constants in the multi-laver }\end{array}$ \\
\hline$E_{\mathrm{f}}$ & fibre Young's modulus & $A, B, C, D$ & $\begin{array}{l}\text { unknown constants in the multi-layer } \\
\text { model }\end{array}$ \\
\hline$E_{\mathrm{m}}$ & matrix Young's modulus & & $\begin{array}{l}\text { model } \\
\text { fibre angle of inner tape laver }\end{array}$ \\
\hline$E_{1}$ & stiffness in cord direction & $\theta_{1}$ & $\begin{array}{l}\text { fibre angle of inner tape layer } \\
\text { fibre angle of outer tape laver }\end{array}$ \\
\hline$\nu_{\mathrm{m}}$ & matrix Poisson’s ratio & $\theta_{2}$ & $\begin{array}{l}\text { fibre angle of outer tape layer } \\
\text { initial fibre angle }\end{array}$ \\
\hline$\rho_{\mathrm{c}}$ & linear cord density & $\theta_{0}$ & \\
\hline$\rho_{\mathrm{f}}$ & fibre density & {$[Q]$} & stiffness matrix for unidirectional ply \\
\hline$h_{\mathrm{f}}$ & thickness fibre layer used in model & {$[Q]$} & $\begin{array}{l}\text { stiffness matrix for pipe wall or trans- } \\
\text { formed reduced stiffness matrix }\end{array}$ \\
\hline & internal pressure & {$[T]$} & transformation matrix \\
\hline$\theta \theta, \varepsilon_{z z}, \gamma$ & strams in gioval directions & {$[R]$} & Reuter's matrix \\
\hline
\end{tabular}

and the matrix. This results in a low flexural stiffness of the pipe, so that the pipe can be reeled. The liner pipe and cover layer are of the same generic type of thermoplastic material, heat bonded together providing a sturdy monolithic construction. There is about $200 \mathrm{~km}$ of this long length RTP, hereafter called long length reinforced thermoplastic pipe (LLRTP), in operation now.

In order to design and engineer LLRTPs, it is very important to have a clear understanding of the mechanical behaviour of LLRTPs. For this purpose, a procedure to predict the resulting stresses and strains as a result of a particular loading case is needed. Mathematical models to predict the stresses and strains for LLRTPs were already reported in Refs. [2,4]. However, presented in a different way, the models in Refs. [2,4] are essentially the same. Both models assume a plane stress state, with no load take up in the direction perpendicular to the fibres. The measured and calculated strains in axial and hoop direction were presented as a function of the internal pressure. The apparent mismatch between the calculated and the measured hoop strain at low pressures was found to be a result of the cordslack. Cord-slack is a certain surplus length of the cords relative to the pipe geometry. This phenomenon will be discussed in more detail later on. The hoop strain for higher pressures was well described by both models. The models did not show an adequate agreement with the experimentally determined axial strain. A discussion on the torsional behaviour was absent in both cases. However, peculiar torsional behaviour was observed for the current LLRTP when pressurised, as the rotation direction sudden changes sign at about 35 bar. This behaviour needs to be analysed and described. The current study can be seen as an extension of the models mentioned above. The focus is set on predicting the torsional behaviour as well as the tangential and axial strains of the LLRTP when loaded by hydrostatic pressure. First, the materials used and the production process are outlined. As it is observed that the amount of cord-slack will be different for both layers, a procedure to measure the amount of cord-slack for the two different layers is described. After that, a model for the torsional behaviour is developed by incorporating the different slack values into a modified version of the existing plane stress model $[2,4]$. Finally, an updated model for LLRTP based on a plain strain characterisation will be introduced. In the subsequent sections of this paper this model will be referred to as the multi-layer model.

\section{LLRTP manufacturing method}

\subsection{Pipe materials}

The thermoplastic resin, which is used for the reinforcement tapes, the liner and the cover, is a pipe grade HDPE, type PE100 (Eltex TUB 121), which is hereafter called PE. The main reasons for choosing this material are its long-standing record in the oil and gas industry. Some technical details on

Table 1

Physical and mechanical properties of PE100

\begin{tabular}{|c|c|c|c|c|}
\hline Property & Symbol & Standard & Unit & Value \\
\hline $\begin{array}{l}\text { Density } \\
\text { (compound) }\end{array}$ & $\rho_{\mathrm{m}}$ & ISO/R 1183 & $\mathrm{~g} / \mathrm{cm}^{3}$ & $0.9600-0.9609$ \\
\hline $\begin{array}{l}\text { Yield stress } \\
(100 \mathrm{~mm} / \mathrm{min})\end{array}$ & $\sigma_{\mathrm{y}}$ & $\begin{array}{l}\text { ISO/DIS } \\
6259,2\end{array}$ & $\mathrm{MPa}$ & $>25$ \\
\hline $\begin{array}{l}\text { Young's- } \\
\text { modulus }\end{array}$ & $E_{\mathrm{m}}$ & ISO $527-2$ & $\mathrm{MPa}$ & 1205 \\
\hline Poisson's ratio & $\nu_{\mathrm{m}}$ & & & 0.4 \\
\hline $\begin{array}{l}\text { Elongation } \\
\text { at break } \\
(100 \mathrm{~mm} / \mathrm{min})\end{array}$ & $\varepsilon_{\max }$ & $\begin{array}{l}\text { ISO/DIS } \\
6259,2\end{array}$ & $\%$ & $>600$ \\
\hline
\end{tabular}




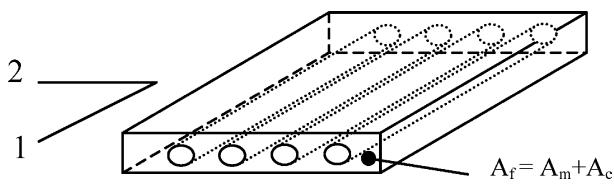

Fig. 1. Outline of the aramid reinforced tape with the definition of the local co-ordinate system used for the theoretical model. $A_{\mathrm{f}}, A_{\mathrm{m}}$ and $A_{\mathrm{c}}$ represent the cross-sections of the tape, the matrix material and the aramid cords, respectively.

PE100 are given in Table 1. The stress-strain relationship for $\mathrm{PE}$ is non-linear and depends on the strain and the strain-rate. In this study, the PE is modelled as a linear elastic material, using a secant modulus of $400 \mathrm{MPa}$. This value is based on the loading speed, temperature and average strain during the LLRTP pressurisation experiments. For the reinforced tapes the same PE is used. The cords are embedded in, but not impregnated by, the PE. The Twaron ${ }^{\circledR}$-aramid cords itself are twisted in order to avoid filament buckling when the cords are subjected to a negative strain. For the model, a measured stiffness of $80 \mathrm{GPa}$ is used for the cords. The reinforcing tapes, from which an outline is shown in Fig. 1, are produced by an extrusion process. In Fig. $1, A_{\mathrm{f}}, A_{\mathrm{m}}$ and $A_{\mathrm{c}}$ are the total cross-section area, the cross-section area of the matrix and the cross-section area of the cords, respectively. The fibre direction and the in-plane direction perpendicular to the fibres, denoted the ' 1 ' and ' 2 ' direction, respectively, are called the principal directions. The use of non-impregnated cords in a tape is not conventional and probably viable only with aramid, because of its high abrasion resistance.

\subsection{Helical tape wrapping method}

Commercially available LLRTP is commonly produced by the helical tape wrapping method. An important advantage of this process is that it is continuous. First, the HDPE liner is extruded and cooled immediately. In the second stage, the first tape layer is wrapped over the extruded liner. The resulting winding angle is fixed to $54^{\circ}$ by the outer diameter of the liner and the tape width. As indicated in Fig. 2, the winding tension involved is balanced by wrapping two tapes per pitch at the same time. The procedure for wrapping the second layer will be exactly the same, only the wrapping angle will be $-54^{\circ}$. It must be noted that because of logistic reasons the same tape width is used for both layers, resulting in a slight increase (about $1.3^{\circ}$ ) of the winding angle. The three components are heat bonded together after wrapping the two tape layers. The structure is cooled rapidly after the heat bonding procedure and a cover layer is extruded over it. Finally the LLRTP is cooled again, printed and reeled.

\subsection{Cord-slack and the influences on the mechanical properties}

As already described in Refs. [2,4], the cords will not immediately contribute to the stiffness of the LLRTP. A certain strain, which is called the 'take-up strain', is needed to pull taut the twisted aramid cords. This phenomenon of the cords having a certain surplus length to the pipe geometry is called cord-slack. The total amount of cordslack for the cords in the pipe is the result of two separate mechanisms. The first part is the construction strain of the twisted aramid cords. This amount of construction strain is equal for both layers. The second part can be imputed to shrinkage related geometrical changes during and after the production process. Unlike to the first mechanism, the effect of this shrinkage related part is not equal for both fibre layers, since these are wrapped under a slightly different

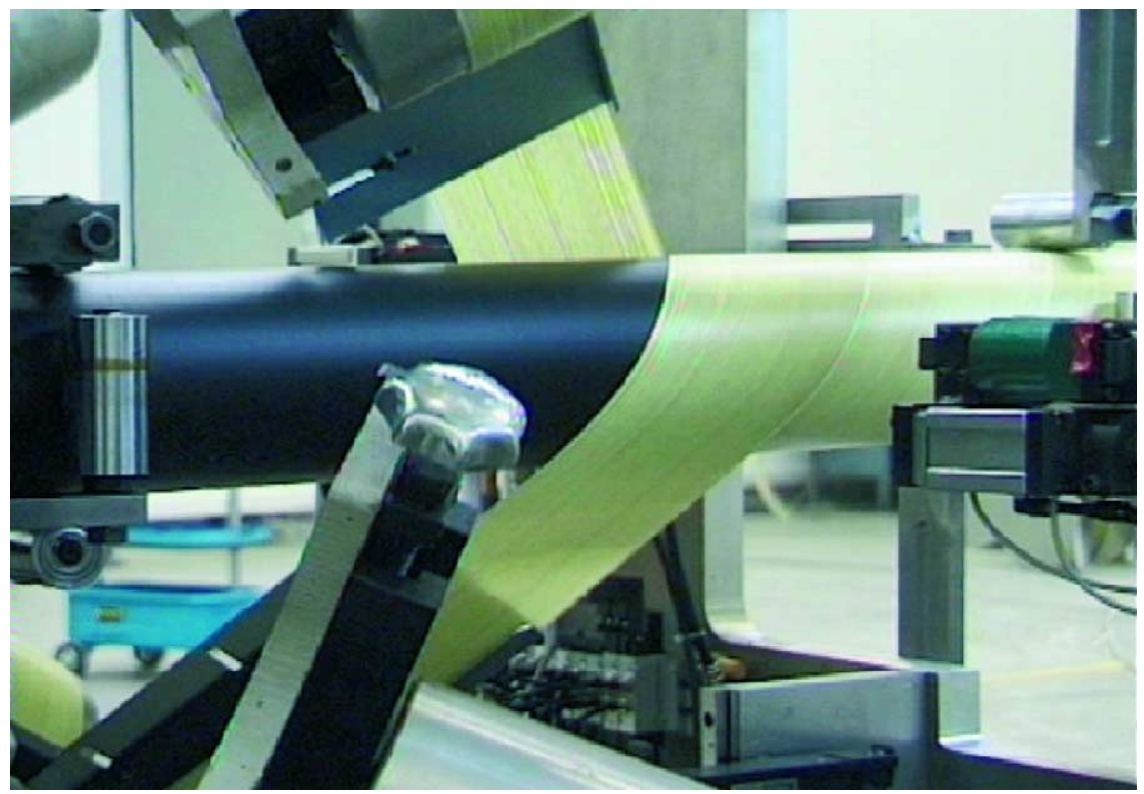

Fig. 2. The wrapping process for fibre layer 1. (picture Krauss Maffei Kunsstofftechnik). 


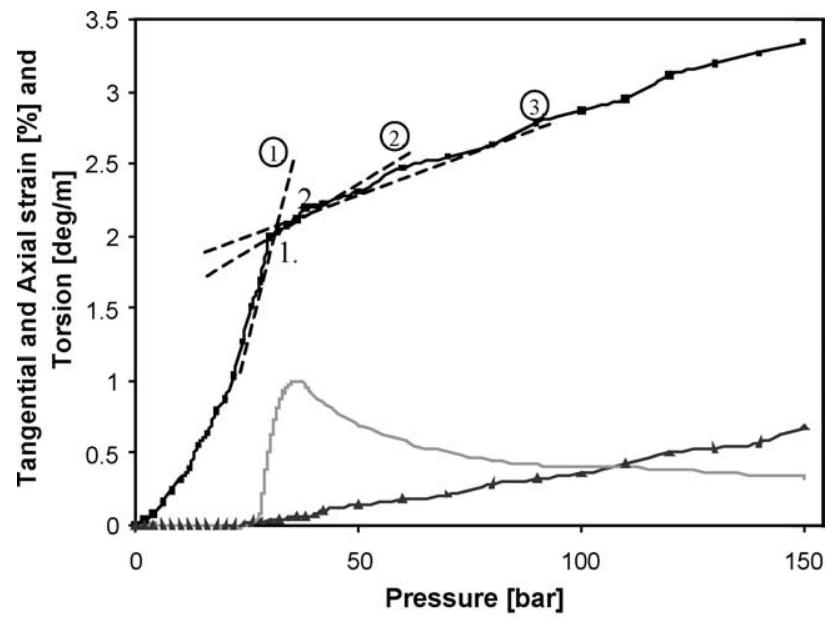

$\rightarrow$ tangential strain $\quad \longleftarrow$ axial strain $\quad$ torsion

Fig. 3. Typical behaviour of a 4 " pressurised LLRTP. The circled numbers 1,2 and 3 represent the three different values for the tangential compliance indicated when subjecting an RTP to internal pressure. The non-circled numbers 1 and 2, which are used for the cord-slack estimations procedure, are defined by the intersections of line 1 and 2 and line 2 and 3 , respectively.

angle. A 'slack difference' between the two layers is therefore induced. An example of the results of deformation measurements with a $4^{\prime \prime}$ (inner diameter) pressurised LLRTP are shown Fig. 3. The influence of the cord-slack is most obviously presented by the tangential strain. Within the pressure range of $0-150$ bar three different values of tangential compliance can be derived. They are indicated in Fig. 3 by the circled numbers 1, 2 and 3 . From 0 to \pm 30 bar the two fibre layers are not contributing to any load take up. Within this range the LLRTP behaves as a plain PE pipe. At \pm 30 bar, the slack in one of the fibre layers is consumed, and the fibre layer starts to take up load. This is confirmed by the torsional behaviour of the pipe, which starts at the same time. It can be demonstrated by laminate theory or by the simple fact, the fibre layer will tend to unwind itself, the jump in torsion angle is caused by the outer fibre layer. Because of the slack difference, the inner fibre layer does not directly resist the torsion moment generated by the outer fibre layer. At \pm 35 bar the slack in the inner fibre layer is also consumed due to the torsion angle and the increasing tangential and axial strain. This results in equal torsion moments generated by the two fibre layers at this point. When the pressure is further increased, the LLRTP shows rotation in the opposite direction. This is a result of the unbalanced torsion moments generated by the two fibre layers, which will be treated in more detail later in this paper.

\section{Experimental procedures}

\subsection{Pressure experiments}

This study is concerned with one type of test, in which the pipe is only subjected to an internal pressure. The LLRTP samples were $2 \mathrm{~m}$ long and terminated at both ends by specially designed end fittings [5]. In each case, the pressure loading rate was $15 \mathrm{bar} / \mathrm{min}( \pm 5 \%)$. A dedicated pipe testing machine (Flexi-rig) was used for the pressure experiments, as shown in Fig. 4. The Flexi-rig can subject pipes to arbitrary combinations of internal pressure, torsion moment and axial forces. The resulting displacements in axial direction and the torsion angle can be measured simultaneously. A specially designed linear variable differential transformer (LVDT) device was used [6] for the hoop displacement and an extra measurement of the axial displacement directly on the pipe. The LLRTP should be free to deform during the pressure experiment, therefore force control was used by continuously driving the resulting axial forces and torsion moments to zero. The resulting axial displacement $w$ and the torsion angle $\theta$ of the movable head

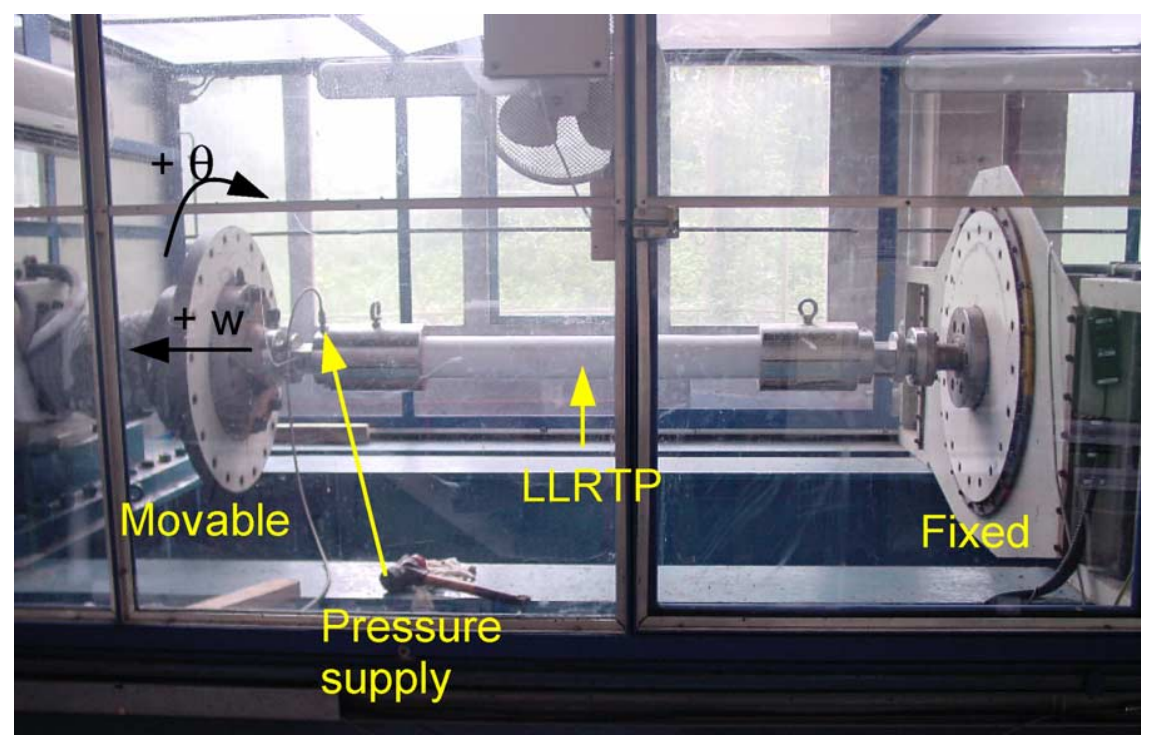

Fig. 4. LLRTP fixed in the Flexi-rig during a hydrostatic pressure experiment. 


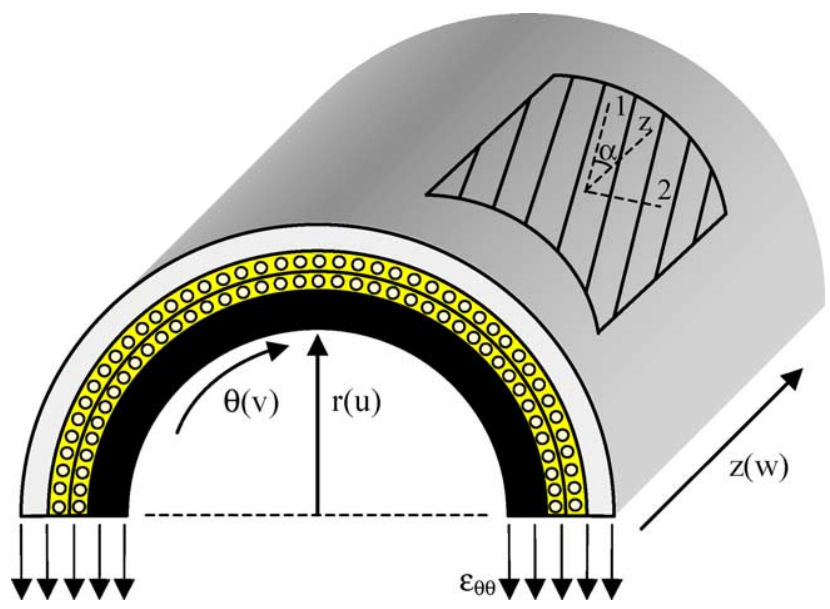

Fig. 5. Plane stress cross-section of an LLRTP with the definition of the global $(r, \theta, z)$ and the local $(1,2)$ coordinate system. The cord angle $\alpha$, is defined by the angle between the (local) 1-direction and the (global) $z$-direction. $\varepsilon_{\theta \theta}$ represents the tangential strain, which is here assumed to be homogeneous through the thickness.

were measured, together with the tangential and axial displacements measured directly on the pipe by the LVDTdevice.

\subsection{Cord-slack estimation}

In this procedure, the amount of the take-up strain is estimated, which is needed to pull taut the cords in the fibre layers, for two models further to be specified. This is done by examining the strains in the global directions $r, z$ and $\theta$ (see Fig. 5) at critical points 1 and 2, which are defined by the intersections of line 1 and 2 and line 2 and 3 , respectively (Fig. 3). For the plane stress model, the strains in the global directions in the fibre layers are equal to the strains measured at the outside of the cylinder. The global strains can be translated to the strain in the cord direction in the fibre layers by Eq. (1). When the global strains at critical points 1 and 2 (Fig. 3) are substituted in Eq. (1), the amount of cord-slack is found for fibre layers 2 and 1 , respectively

$\varepsilon_{1}=\varepsilon_{\theta \theta} \cos ^{2} \alpha+\varepsilon_{z z} \sin ^{2} \alpha+\gamma_{z \theta} \sin \alpha \cos \alpha$,

where $\varepsilon_{1}$, the strain in the cord direction, is the amount of cord-slack in this case and $\alpha$ is the angle between the fibre direction and the $z$-axis. For the multi-layer model, where the strains (except for $\varepsilon_{z z}$ ) are a function of the pipe radius $r$, the strains measured at the outside of the cylinder need first to be translated to the strains at the fibre layers, before applying Eq. (1). The resulting cord-slack parameters or

Table 2

Estimated cord-slack values

\begin{tabular}{lll}
\hline & $\begin{array}{l}\text { Plane stress } \\
\text { model }(\%)\end{array}$ & $\begin{array}{l}\text { Multi-layer } \\
\text { model }(\%)\end{array}$ \\
\hline Fibre layer 1 & 1.6 & 1.75 \\
Fibre layer 2 & 1.45 & 1.6 \\
\hline
\end{tabular}

take-up strain needed in the cord direction to load the fibres are given in Table 2 .

\section{Modelling}

In this section, the existing plane stress model will first be extended by incorporating the non-linear behaviour as a result of the change in fibre angles and internal diameter during pressurisation. After that a new model for LLRTP, based on a plane strain characterisation will be introduced.

\subsection{Updated 'plane stress' model}

For this simple approach, the stresses and strain do not depend on co-ordinates $r, z$ and $\theta$ and thus the stresses $\sigma_{z z}$, $\sigma_{\theta \theta}$ and $\sigma_{z \theta}$ (disregarding any radial stresses) within one layer are uniform through the thickness. First the stressstrain relationships will be presented, which follow from general laminate theory presented in Ref. [7]. After that, these relationships are applied in a modified form for the solution of this particular problem. The stresses and strains for the isotropic and the fibre layers are denoted by superscript ' $m$ ' and ' $\mathrm{f}$ ', respectively throughout this section.

When plane stress is assumed the relation between the stresses and strains for an isotropic layer can be represented by

$$
\left(\begin{array}{c}
\sigma_{\theta \theta}^{\mathrm{m}} \\
\sigma_{z z}^{\mathrm{m}} \\
\sigma_{z \theta}^{\mathrm{m}}
\end{array}\right)=\left[\begin{array}{ccc}
Q_{11} & Q_{12} & 0 \\
Q_{12} & Q_{22} & 0 \\
0 & 0 & Q_{66}
\end{array}\right] \cdot\left(\begin{array}{c}
\varepsilon_{\theta \theta}^{\mathrm{m}} \\
\varepsilon_{z z}^{\mathrm{m}} \\
\varepsilon_{z \theta}^{\mathrm{m}}
\end{array}\right),
$$

where the stiffness matrix $[Q]$ contains the elastic constants of the isotropic material. The definitions of the different elements of the [Q] matrix can be found in Ref. [7].

For the unidirectional tape layers, loaded in-plane along its principal directions, the relation between the stresses and strains can be represented by

$$
\left(\begin{array}{c}
\sigma_{1}^{\mathrm{f}} \\
\sigma_{2}^{\mathrm{f}} \\
\sigma_{12}^{\mathrm{f}}
\end{array}\right)=\left[\begin{array}{ccc}
Q_{11} & Q_{12} & 0 \\
Q_{12} & Q_{22} & 0 \\
0 & 0 & Q_{66}
\end{array}\right] \cdot\left(\begin{array}{c}
\varepsilon_{1}^{\mathrm{f}} \\
\varepsilon_{2}^{\mathrm{f}} \\
\varepsilon_{12}^{\mathrm{f}}
\end{array}\right)
$$

It must be noted that although the relation's (2) and (3) are identical, the elements of the $[Q]$ matrix in Eq. (3) are defined in another way [7] and have different values in different directions. When the unidirectional tape layer is stressed in the global directions $(z, \theta)$, which are at an angle $\alpha$ to the principal directions $(1,2)$, the resulting strains can be evaluated by the following relation

$$
\left(\begin{array}{c}
\sigma_{\theta \theta}^{\mathrm{f}} \\
\sigma_{z z}^{\mathrm{f}} \\
\sigma_{z \theta}^{\mathrm{f}}
\end{array}\right)=\left[Q^{*}\right] \cdot\left(\begin{array}{c}
\varepsilon_{\theta \theta}^{\mathrm{f}} \\
\varepsilon_{z z}^{\mathrm{f}} \\
\gamma_{z \theta}^{\mathrm{f}}
\end{array}\right)
$$


where $\left[Q^{*}\right]$ is a fully populated $[3 \times 3]$ matrix, relating the stresses to the strains in global directions for an unidirectional fibre layer under in-plane off-axis loading. The $\left[Q^{*}\right]$ matrix is defined by

$\left[Q^{*}\right]=[T(\alpha)]^{-1} \cdot[Q] \cdot[R] \cdot[T(\alpha)] \cdot[R]^{-1}$,

where

$[T(\alpha)]=\left(\begin{array}{ccc}\cos ^{2} \alpha & \sin ^{2} \alpha & 2 \sin \alpha \cos \alpha \\ \sin ^{2} \alpha & \cos ^{2} \alpha & -2 \sin \alpha \cos \alpha \\ -\sin \alpha \cos \alpha & \sin \alpha \cos \alpha & \cos ^{2} \alpha-\sin ^{2} \alpha\end{array}\right)$

and

$[R]=\left(\begin{array}{lll}1 & 0 & 0 \\ 0 & 1 & 0 \\ 0 & 0 & 2\end{array}\right)$

For the case considered here, the stress-strain relations for the unidirectional reinforced tape can be simplified. For the model, the height of the reinforced tape is taken equal to the diameter of the aramid cords. When it is assumed that the cords are perfectly cylindrical the height of the unidirectional fibre layer $h_{\mathrm{f}}$ is defined as

$h_{\mathrm{f}}=\sqrt{\frac{4 \rho_{\mathrm{c}}}{\pi \rho_{\mathrm{f}}}}$

where $\rho_{\mathrm{c}}$ is the cord linear density, commonly expressed in dtex and $\rho_{\mathrm{f}}$ is the fibre density. The PE of the reinforced tape, which is located under and above the cords, indicated by the dotted area in Fig. 6 (right), is attributed to the isotropic layers. As there is no adhesive bonding between the PE and the cords, no stresses can be transferred in the principal 2-direction of the reinforced tape. As a result, there is only a connection between $\sigma_{1}$ and $\varepsilon_{1}$ in Eq. (3). The values for $Q_{12}, Q_{22}$ and $Q_{66}$ in Eq. (3) are equal to zero. To determine $Q_{11}$, the stiffness in the 1-direction of the unidirectional fibre layer is needed, which is defined by the rule of mixtures and is equal to

$E_{1}=E_{\mathrm{f}} \cdot V_{\mathrm{f}}+E_{\mathrm{m}}\left(1-V_{\mathrm{f}}\right)$,

where $E_{\mathrm{f}}$ is the fibre modulus and $V_{\mathrm{f}}$ is the fibre volume fraction.

As plane stress is assumed, the stresses for the isotropic layers (i.e. liner and cover) can be represented by three unknown constants

$\sigma_{\theta \theta}^{\mathrm{m}}=A_{1} \quad \sigma_{z z}^{\mathrm{m}}=B_{1} \quad \sigma_{z \theta}^{\mathrm{m}}=C_{1}$

\section{0}

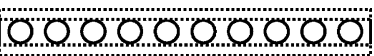

Fig. 6. Cross-section of the tape (left) and the modification used in the model (right). The circles represent the aramid cords. For the modification, the tape height is set equal to the diameter of the cords. The remainder, (dotted area) consisting of plain PE is attributed to the isotopic PE layers in the pipe cross-section (i.e. liner and cover).
As the isotropic and the fibre layers are heat welded together, it further applies that

$$
\left(\begin{array}{c}
\varepsilon_{\theta \theta}^{\mathrm{m}} \\
\varepsilon_{z z}^{\mathrm{m}} \\
\varepsilon_{z \theta}^{\mathrm{m}}
\end{array}\right)=\left(\begin{array}{c}
\varepsilon_{\theta \theta}^{\mathrm{f}} \\
\varepsilon_{z z}^{\mathrm{f}} \\
\gamma_{z \theta}^{\mathrm{f}}
\end{array}\right)
$$

The stresses and strains in the global directions for the isotropic and the fibre layers can be written as a function of the unknown $A_{1}, B_{1}$ and $C_{1}$ by Eqs. (2)-(9). The three unknowns $A_{1}, B_{1}$ and $C_{1}$ in Eq. (8) are finally solved by evaluating the following three equations [4].

(1) Equilibrium of axial forces $\sum F_{z z}=0$

(2) Equilibrium of tangential forces $\sum F_{\theta \theta}=0$

(3) Torque equilibrium $\sum M=0$

The plane stress model is further extended by taking into account the geometrical non-linearities. As a result of the deformations, which are considerable because of the fibre slack, the change of the internal volume as well as the change of the fibre angles are no longer negligible. The fibre angle can be found to vary according to

$\alpha=\alpha_{0}+\left(-\varepsilon_{z z}+\varepsilon_{\theta \theta}\right) \sin \alpha_{0} \cos \alpha_{0}+\gamma_{z \theta} \cos ^{2} \alpha_{0}$,

with $\alpha_{0}$ as the fibre angle in the unloaded state. Substituting expression (11) into Eq. (5) leads to a non-linear system. The cord-slack leads to further non-linearities. Details can be found in Section 4.2. The resulting system is solved iteratively, using a Newton-Raphson method [8].

\subsection{Multi-layer 'generalised plane strain' model}

The previous assumption of uniform stresses through the thickness of one layer is only valid for thin walled tubes. The ratio between the axial and the tangential wall stresses as a result of the hydrostatic pressure is 1:2 in that case. Based on the dimensions the LLRTP it can be demonstrated that this ratio will be equal to $1: 2.25$ rather than $1: 2$, and the stresses can be non-uniform through the thickness. For this reason, the LLRTP will be now considered as a thick cylinder and the stress situation will be characterised as generalised plane strain. The deformation in the $z$ direction is assumed to be uniform. The stresses and strains (except for $\varepsilon_{z z}$ and $\sigma_{z z}$ ) will be no longer uniform, but they will depend on the $r$-co-ordinate. First, the stress functions will be deduced from general mechanics for this new approach. This is presented by Eqs. (12)-(18). After that, the unknown constants are solved by formulating boundary conditions for this particular pipe problem.

The stresses must satisfy the equilibrium equations in cylindrical co-ordinates, in the absence of body forces 
$\frac{\partial \sigma_{r r}}{\partial r}+\frac{1}{r} \cdot \frac{\partial \sigma_{\theta r}}{\partial \theta}+\frac{\partial \sigma_{z r}}{\partial z}+\frac{\sigma_{r r}-\sigma_{\theta \theta}}{r}=0$

$\frac{\partial \sigma_{r \theta}}{\partial r}+\frac{1}{r} \cdot \frac{\partial \sigma_{\theta \theta}}{\partial \theta}+\frac{\partial \sigma_{z \theta}}{\partial z}+\frac{2 \cdot \sigma_{\theta r}}{r}=0$

$\frac{\partial \sigma_{r z}}{\partial r}+\frac{1}{r} \cdot \frac{\partial \sigma_{\theta z}}{\partial \theta}+\frac{\partial \sigma_{z z}}{\partial z}+\frac{\sigma_{r z}}{r}=0$

Assuming axi-symmetry and generalised plane strain, the equations in Eq. (12) can be simplified to

$\frac{\partial \sigma_{r r}}{\partial r}+\frac{\sigma_{r r}-\sigma_{\theta \theta}}{r}=0 \quad \frac{\partial \sigma_{r \theta}}{\partial r}+\frac{2 \cdot \sigma_{r \theta}}{r}=0$

The strain-displacement relations are in this case

$$
\begin{array}{ll}
\varepsilon_{r r}=\frac{\partial u}{\partial r} & \gamma_{r \theta}=\gamma_{\theta r}=\frac{\partial v}{\partial r}-\frac{v}{r} \\
\varepsilon_{\theta \theta}=\frac{u}{r} & \gamma_{\theta z}=\gamma_{z \theta}=\frac{\partial v}{\partial z} \\
\varepsilon_{z z}=\frac{\partial w}{\partial z}=\text { Const. } & \gamma_{z r}=\gamma_{r z}=0
\end{array}
$$

For isotropic linear elastic material, the constitutive equations are given by

$\sigma_{r r}=\lambda\left(\varepsilon_{r r}+\varepsilon_{\theta \theta}+\varepsilon_{z z}\right)+2 \cdot \mu \cdot \varepsilon_{r r}$

$\sigma_{\theta \theta}=\lambda\left(\varepsilon_{r r}+\varepsilon_{\theta \theta}+\varepsilon_{z z}\right)+2 \cdot \mu \cdot \varepsilon_{\theta \theta}$

$\sigma_{z z}=\lambda\left(\varepsilon_{r r}+\varepsilon_{\theta \theta}+\varepsilon_{z z}\right)+2 \cdot \mu \cdot \varepsilon_{z z} \quad \sigma_{z \theta}=\mu \cdot \gamma_{z \theta}$

where $\lambda$ and $\mu$ are the Lame constants. When the strains (14) are substituted into the constitutive equations (15) and the resulting stresses into the second simplified stress equilibrium equation (13) it can be found (see Appendix A) that

$\sigma_{z \theta}=B \cdot r$,

where $B$ is an unknown constant. With the use of Eqs. (13)-(15) it can be found that the stresses in radial and tangential directions can be expressed as

$\sigma_{r r}=\frac{-A}{r^{2}}+C \quad \sigma_{\theta \theta}=\frac{A}{r^{2}}+C$,

where $A$ and $C$ are unknown constants. By substituting Eq. (17) into Eq. (15), it can be found that the stress in the $z$-direction does not depend on $r$ and $z$

$\sigma_{z z}=D$,

where $D$ is an unknown constant to be determined. Since the cords are very thin compared to the pipe it is assumed that the stresses and strains can be modelled as uniform through the thickness and thus plane stress is assumed for the fibre layers. As indicated in Fig. 7, it is assumed that the LLRTP consists of three layers of isotropic linear elastic PE, which are separated by the two infinitely thin fibre layers. The three isotropic layers are defined by

$l_{i}=R_{2 i-1}-R_{2 i-2} \quad$ for $i=1$ to 3

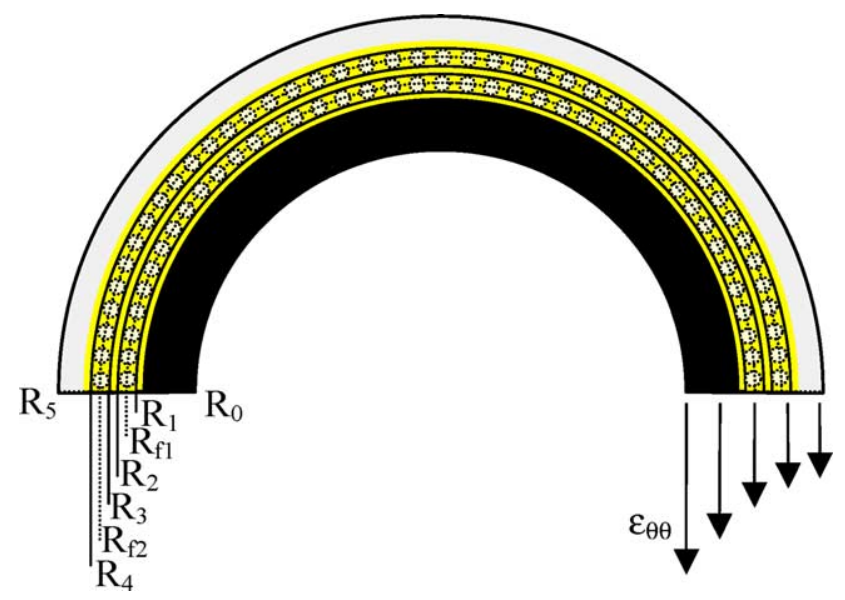

Fig. 7. Dimensions used for multi-layer model.

It is assumed, as in the previous described model that the strains in the fibre layers are equal to the strains in the isotropic material. For each of the three layers, the unknown constants $A, B, C$ and $D$ have to be solved, which brings the total amount of unknown constants to 12. The constants can be determined from conditions on the interfaces of the different layers supplemented with similar equilibrium considerations as stated in Eq. (10). First of all, continuity of the circumferential displacement $v$, which is derived in Appendix A, implies that the constant $B$ has to be equal for each layer. This reduces the amount of unknown constants to 10 . The necessary conditions to solve the remaining 10 constants for pressures larger than the second critical pressure point are given below.

- Interface conditions for the radial strains

$$
\begin{aligned}
& \varepsilon_{r r}^{11}=\varepsilon_{r r}^{12} \quad \text { for } \quad r=R_{\mathrm{f} 1} \quad \text { and } \\
& \varepsilon_{r r}^{12}=\varepsilon_{r r}^{13} \quad \text { for } \quad r=R_{\mathrm{f} 2},
\end{aligned}
$$

where $R_{\mathrm{f} 1}$ and $R_{\mathrm{f} 2}$ are the radii for the position of fibre layer 1 and 2, respectively (see also Fig. 7).

- Interface conditions for the axial strains

$$
\begin{aligned}
& \varepsilon_{z z}^{11}=\varepsilon_{z z}^{12} \quad \text { for } \quad r=R_{1} \text { to } R_{5} \quad \text { and } \\
& \varepsilon_{z z}^{12}=\varepsilon_{z z}^{13} \quad \text { for } \quad r=R_{1} \text { to } R_{5}
\end{aligned}
$$

- Edge stress conditions

$$
\begin{aligned}
& \sigma_{r r}^{11}=-P \quad \text { for } \quad r=R_{0} \quad \text { and } \\
& \sigma_{r r}^{13}=0 \quad \text { for } \quad r=R_{5}
\end{aligned}
$$

The infinitely thin fibre layers will cause discontinuities in the radial and the tangential stress from one isotropic layer to another. The discontinuities in the radial stresses should balance the tangential stresses in the cords by 
the following expressions

$$
\begin{aligned}
& \frac{2 \pi R_{\mathrm{f} 1}^{2}}{\tan \left(\theta_{1}\right)} \cdot\left(\sigma_{r r}^{12}-\sigma_{r r}^{11}\right)=\sigma_{\theta \theta}^{\mathrm{f} 1} \cdot \frac{A_{\mathrm{f}}}{\sin \left(\theta_{1}\right)}-F_{\theta \theta 1}^{\mathrm{s}} \quad \text { and } \\
& \frac{2 \pi R_{\mathrm{f} 2}^{2}}{\tan \left(\theta_{2}\right)} \cdot\left(\sigma_{r r}^{13}-\sigma_{r r}^{12}\right)=\sigma_{\theta \theta}^{\mathrm{f} 2} \cdot \frac{A_{\mathrm{f}}}{\sin \left(\theta_{2}\right)}-F_{\theta \theta 2}^{\mathrm{s}},
\end{aligned}
$$

where the terms with superscript s are the slack terms and $F$ indicates the force exerted by the cords. The slack terms are defined and treated in more detail below.

- Torque equilibrium

$$
\sum_{i=1}^{2} \frac{A_{\mathrm{f}} R_{\mathrm{f} i} \sigma_{z \theta}^{\mathrm{f} i}}{\cos \left(\theta_{i}\right)}-F_{z \theta}^{\mathrm{s}}+\sum_{i=1}^{3} \int_{R_{2 i-2}}^{R_{2 i-1}} r \sigma_{z \theta} 2 \pi r \mathrm{~d} r=0
$$

where $\sigma_{z \theta}$ is given in Eq. (16).

- Equilibrium of axial forces

$$
\sum_{i=1}^{3} \pi\left(R_{2 i-2}^{2}-R_{2 i-1}^{2}\right) \cdot \sigma_{z z}^{1 i}+\sum_{i=1}^{2} \frac{A_{\mathrm{f}} \sigma_{z z}^{\mathrm{f} i}}{\cos \left(\theta_{i}\right)}-F_{z z}^{\mathrm{s}}=P \pi R_{0}^{2}
$$

The cord-slack $\varepsilon^{\mathrm{s}}$ is the estimated slack in the cord direction. The resulting stress in the cords is equal to the cord stiffness times the strain minus the cord-slack, which is expressed as a compressive strain. This is done by subtracting the terms: $F_{\theta \theta}^{\mathrm{s}}, F_{z \theta}^{\mathrm{s}}$ and $F_{z z}^{\mathrm{s}}$ from the resulting fibre layer forces. These three terms are defined as

$F_{\theta \theta i}^{\mathrm{s}}=V_{\mathrm{f}} A_{\mathrm{f}} E_{\mathrm{f}} \varepsilon_{\mathrm{f} i}^{\mathrm{s}} \sin \left(\theta_{i}\right) \quad$ for $i=1,2$

$F_{z \theta}^{\mathrm{s}}=\sum_{i=1}^{2} V_{\mathrm{f}} A_{\mathrm{f}} E_{\mathrm{f}} \varepsilon_{\mathrm{f} i}^{\mathrm{s}} \sin \left(\theta_{i}\right) R_{\mathrm{f} i}$

$F_{z z}^{\mathrm{s}}=\sum_{i=1}^{2} V_{\mathrm{f}} A_{\mathrm{f}} E_{\mathrm{f}} \varepsilon_{\mathrm{f} i}^{\mathrm{s}} \cos \left(\theta_{i}\right)$

Again the same non-linearities as described for the plane stress model are taken into account. The constants are found solving the equations using a Newton-Raphson method, after which all strains, stresses and cord forces can be calculated.

\section{Results}

The results of the experiment and the plane stress model, are presented in Fig. 8. As already mentioned, the pipe is only subjected to an internal pressure and is free to deform in all directions. The input data, which is used for the model can be found in Table 3. The tangential strain is well predicted for pressures larger than 35 bar. The deviations between the measured and the modelled tangential strains at lower pressures can be explained by the non-linear behaviour of the PE, whereas the model describes the PE

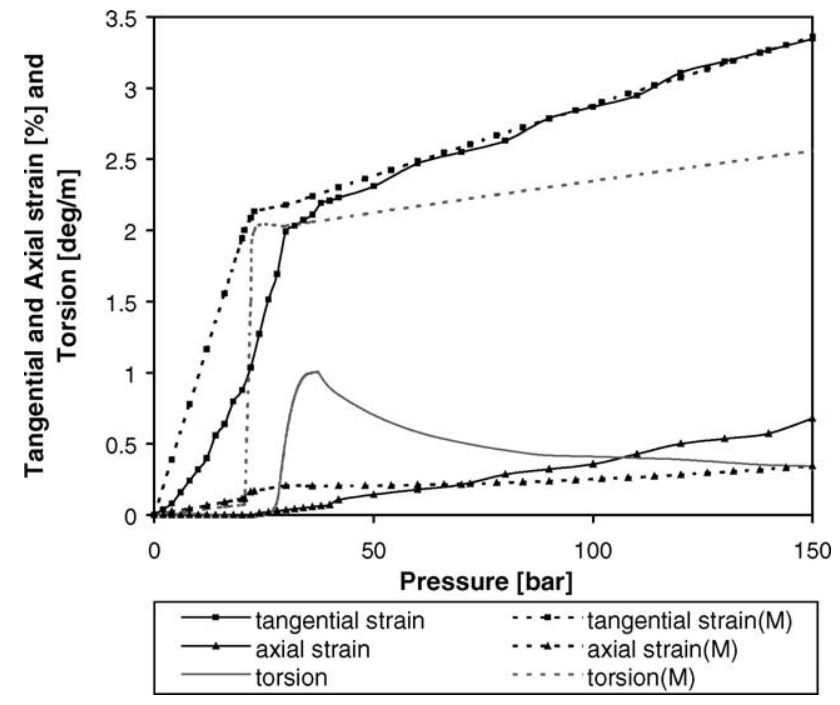

Fig. 8. Comparison of experimental and predicted $(M)$ values for hydrostatic pressure load-case. For the predicted values the updated plane stress model is used. The dotted and the continuous lines refer to the calculated values and the experimental data, respectively.

as a linear elastic material. The prediction of the axial strain is observed to be less accurate. The RTP is modelled too stiff in the axial direction, which cannot be caused by a mismatch of the fibre angles, as the tangential strain is very well described. The torsion angle is not very well predicted either. When the estimated values for the fibre slack are used, the jump in torsion angle is indeed in the right direction but far larger than the experimentally measured jump in torsion angle. For pressures higher than the second critical pressure point, the model predicts a further increase of the torsion angle, while the experiments show the opposite trend.

The results for the multi-layer model are presented in Fig. 9. It is obvious from Fig. 9 that the strains are far better described by the multi-layer model than for the plane stress model. For the axial strain, the largest deviation appears at pressures before the critical pressures, where hardly any axial strain was measured. The model however predicts a linearly increasing axial strain for this pressure range. It is very likely that this deviation can be attributed to the viscoelastic behaviour of the PE. The jump in torsion angle of about $1 \% \mathrm{~m}$ is very well described by the model when the estimated cord-slack difference of $0.15 \%$ is used in the multi-layer model. When the slack is consumed for both layers, the model also predicts a reverse of the torsion angle. It is likely that the more non-linear character of

Table 3

Input data used in model for a 4 " LLRTP

\begin{tabular}{llll}
\hline$R_{0}$ & $50 \mathrm{~mm}$ & $R_{5}$ & $62.5 \mathrm{~mm}$ \\
$R_{1}$ & $56.54 \mathrm{~mm}$ & $R_{\mathrm{f} 1}$ & $57.15 \mathrm{~mm}$ \\
$R_{2}$ & $57.76 \mathrm{~mm}$ & $R_{\mathrm{f} 2}$ & $58.95 \mathrm{~mm}$ \\
$R_{3}$ & $58.34 \mathrm{~mm}$ & $V_{\mathrm{f}}$ & 0.46 \\
$R_{4}$ & $59.56 \mathrm{~mm}$ & $h_{\mathrm{f}}$ & $1.22 \mathrm{~m}$ \\
\hline
\end{tabular}




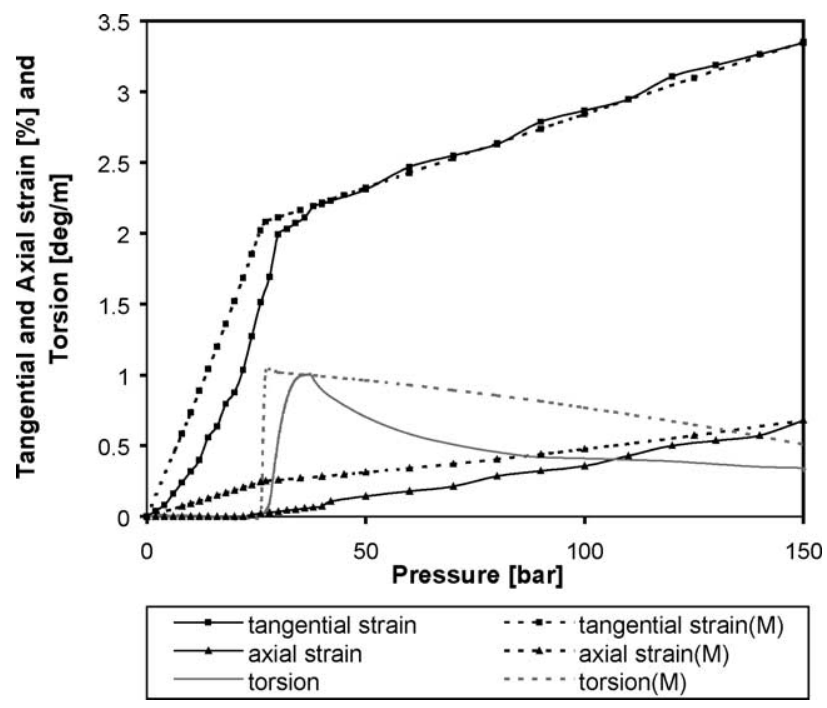

Fig. 9. Comparison of experimental and predicted $(M)$ values for hydrostatic pressure load-case. For the predicted values the multi-layer model is used. The dotted and the continuous lines refer to the calculated values and the experimental data, respectively.

the measured reverse torsion angle is also a result of the visco-elastic behaviour of the PE.

\section{Discussion}

Although the inner and outer fibre layers generate a positive and negative torsion moment respectively, they are not balanced as indicated by the negative torsion angle at values of the inner pressure larger than the second critical pressure point. The resulting torsion moment induced by one layer is defined as the resulting shear force times the distance to the centre of the RTP. Three mechanisms are responsible for the unbalance in torsion moments:

1) The two layers have different fibre angles

$\theta_{1} \neq \theta_{2} \Rightarrow \tau_{z \theta}^{f 1} \neq \tau_{z \theta}^{f 2} \Rightarrow F_{z \theta}^{f 1} \neq F_{z \theta}^{f 2}$

2) The two layers have different fibre radii

$R_{f 1} \neq R_{f 2}$

3) The strains are non-uniform trough the thickness

$\varepsilon_{\theta \theta}^{f 1} \neq \varepsilon_{\theta \theta}^{f 2} \Rightarrow F_{z \theta}^{f 1} \neq F_{z \theta}^{f 2}$

The first two mechanisms are taken into account in the plane stress model and resulted in a monotonously increasing torsion angle. A reduction of the torsion angle is only found by taking the third mechanism into account in the multi-layer model. This behaviour is made possible by the relatively low elasticity modulus of PE. The RTP is therefore sensitive to a small unbalance of the torsion moments caused by the two different fibre layers.

It is of great importance to take into account the change of the fibre angles during deformation. The average initial fibre angle is equal to $54.7^{\circ}$. This is based on thin cylinder theory, from which it follows that the ratio between the tangential and axial stresses is $2: 1$. When it is assumed that the matrix transfers no loads, one can find:

$\frac{\sigma_{\theta \theta}}{\sigma_{z z}}=\frac{\sin ^{2}(\theta) \cdot \sigma_{1}}{\cos ^{2}(\theta) \cdot \sigma_{1}}=\tan ^{2}(\theta)=2 \Rightarrow \theta=54.73^{\circ}$

Based on the initial geometry of the LLRTP, it can be found that the ratio between the average tangential and axial stresses is in the order of 2.25:1. With the use of Eq. (27) it can be found that the average initial angle should be $56.3^{\circ}$. It can be concluded that with the average initial angle of $54.7^{\circ}$, too much stiffness is applied in the axial direction and as a result too little stiffness is applied in the tangential direction. This is partially corrected by the cord-slack. Because the cord-slack is consumed at about $2 \%$ tangential strain of the LLRTP (without any axial strain), the fibre average fibre angle is equal to $55.24^{\circ}$ when it starts contributing to resist the inner pressure. It was also found that by incorporating the change of the fibre angles as a function of the strains in the model, the average fibre angle converges to $56.3^{\circ}$ by increasing the inner pressure. Including the change of the fibre angles during deformation will be even of greater importance for a good prediction of the resulting strains in case of off axis load-cases.

In most cases axial deformation is unwanted. A perfect LLRTP should not increase in length and should not show any torsional behaviour when pressurised. As the mechanical behaviour is now well understood, it is now possible with the use of the model to optimise the initial angles for the two fibre layers in order to keep the axial strain and the torsion angle both equal to zero at a certain pressure level.

\section{Conclusions and future work}

The mechanical behaviour, and in particular the torsional behaviour of a LLRTP was analysed and explained. It appeared that the complex torsional behaviour is a result of the cord-slack difference between the two fibre layers.

The cord-slack values for the two fibre layers were estimated from the strain-internal pressure behaviour. The existing, earlier published plane stress model was modified to take into account the change in cord angle and the change of the geometry. When the estimated cord-slack values were incorporated into the model, the model did not show good agreement with most of the experimental data.

The model was further extended to take into account the non-uniform strain distribution through the thickness. 
The updated model shows good agreement with the experimental data. To further improve the model it will be necessary to take into account the visco-elastic behaviour of the PE, which is the objective for the ongoing research in this field.

\section{Acknowledgements}

Pipelife Nederland B.V., producer of Soluforce ${ }^{\circledR}$ LLRTP made this research possible by making available their test equipment and LLRTP specimens. The authors wish to express their thanks to the company and most especially to Dr L.G.P. Dalmolen and Dr A. Fahrer for their helpful discussions.

\section{Appendix A. Derivation of the stress function for $\sigma_{z \theta}$}

When the strains (14) are substituted in the constitutive equations (15) and the resulting stresses into the second simplified stress equilibrium equation, it can be found that

$\frac{\partial \gamma_{r \theta}}{\partial r}+\frac{2 \cdot \gamma_{r \theta}}{r}=0$

which can also be written as

$\frac{\partial}{\partial r}\left(r^{2} \gamma_{r \theta}\right)=0, \quad$ with solution $\Rightarrow r^{2} \gamma_{r \theta}=f(z)$

It further implies that

$\gamma_{r \theta}=\frac{\partial v}{\partial r}-\frac{v}{r}=r \frac{\partial}{\partial r}\left(\frac{v}{r}\right)$

Substitution of Eq. (A3) into Eq. (A2) leads to

$\frac{v}{r}=-\frac{f(z)}{2 \cdot r^{2}}+g(z) \quad$ or $\quad v=\frac{E(z)}{r}+F(z) \cdot r$

$\gamma_{z \theta}=\partial v / \partial z$ is only a function of $r$ from which follows that $E^{\prime \prime}(z) / r+F^{\prime \prime}(z) \cdot r$ can only be a function of $r$.
And hence

$E^{\prime \prime}(z)=C_{1} \quad$ en $\quad F^{\prime \prime}(z)=C_{2}$,

leading to

$E(z)=C_{1} \cdot z+D_{1} \quad$ en $\quad F(z)=C_{2} \cdot z+D_{2}$

When Eq. (A6) is substituted into Eq. (A4), this yields

$v=C_{1}\left(\frac{z}{r}\right)+D_{1}\left(\frac{1}{r}\right)+C_{2} \cdot z \cdot r+D_{2} \cdot r$

After applying the boundary conditions for the axial symmetric pipe it turns out that

$v(r, z)=C_{2} \cdot z \cdot r$

With the use of Eqs. (14) and (15) it can be finally found that

$\sigma_{z \theta}=B \cdot r$

\section{References}

[1] Frost SR. Glass fibre-reinforced epoxy matrix filament-wound pipes for use in the oil industry. Compos Manufact 1994;5(2):73-82.

[2] Frost SR. The development of RTP for use in the oil industry. In: Composite materials for offshore operations-2. Houston: American Bureau of Shipping; 1999 p. 341-60.

[3] Fisher EH, Gibson AG. Continuous fibre reinforced thermoplastic pipes for transport and distribution of fluids for the oil and gas industries. Plast, Rubber Compos 1999;27(10):447-51.

[4] Chapman BJ, Evans JT, Frost SR, Gibson AG. Mechanical testing of continuous fibre reinforced thermoplastic pipes. In: Proceedings of 11th International Conference on Composites Materials, Australia p. V517-26.

[5] Soluforce ${ }^{\circledR}$ reinforced thermoplastic pipesystem. Commercial brochure, Pipelife Nederland B.V.

[6] Gibson AG, Hicks C, Wright PNH, Fahrer A. Development of glass fibre reinforced polyethylene pipes for pressure applications. Plast, Rubber Compos 2000;29(10):509-19.

[7] Powell PC. In: Engineering with fibre-polymer laminates. London: Chapman \& Hall; 1994.

[8] Verbeke J. The Newton-Raphson method. Int J Math Educ Sci Technol 1995;26(2):177-94. 